\title{
Il Cristo proibito de Curzio Malaparte : une tragédie néoréaliste
}

Il Cristo proibito di Curzio Malaparte: un tragedia neorealista

Malaparte's Il cristo proibito: A Neorealistic Tragedy

Laurent Scotto

\section{OpenEdition}

\section{Journals}

Édition électronique

URL : http://journals.openedition.org/cei/3404

DOI : 10.4000/cei.3404

ISSN : 2260-779X

\section{Éditeur}

UGA Éditions/Université Grenoble Alpes

Édition imprimée

ISBN : 978-2-84310-370-4

ISSN : 1770-9571

\section{Référence électronique}

Laurent Scotto, « // Cristo proibito de Curzio Malaparte : une tragédie néoréaliste », Cahiers d'études italiennes [En ligne], 24 | 2017, mis en ligne le 28 février 2017, consulté le 26 mars 2021. URL : http:// journals.openedition.org/cei/3404; DOI : https://doi.org/10.4000/cei.3404

Ce document a été généré automatiquement le 26 mars 2021.

(c) ELLUG 


\title{
Il Cristo proibito de Curzio Malaparte : une tragédie néoréaliste
}

\author{
Il Cristo proibito di Curzio Malaparte: un tragedia neorealista \\ Malaparte's Il cristo proibito: A Neorealistic Tragedy
}

\section{Laurent Scotto}

$1 \quad$ Il Cristo proibito est le titre du seul film réalisé par Curzio Malaparte. L'écrivain a écrit plusieurs scénarios, a élaboré d'autres projets cinématographiques mais aucun n'a jamais abouti. Pourtant, l'intérêt de Malaparte pour le septième art est ancien puisque dès 1937 il consacrait un numéro entier de la revue Prospettive, dont il fut le fondateur, au cinéma.

2 Le film est l'adaptation du roman éponyme que Malaparte commence à écrire dès 1945 et pour lequel un contrat avait même été signé, chez Bompiani en Italie et chez Denoël en France ${ }^{1}$. L'ouvrage est ensuite transformé en scénario en 1949. Tourné durant l'été 1950 en Toscane, il est projeté dans les salles italiennes en 1951. Il est présenté au Festival de Cannes en 1951 avec trois autres films italiens dont Miracolo a Milano de Vittorio De Sica qui obtient le prix du meilleur film, puis au Festival de Berlin où il remporte l'Ours d'argent pour ses deux acteurs principaux.

3 Malaparte est une personnalité difficile à cerner, souvent insaisissable. Le lieu où l'auteur se révèle le mieux est alors son œuvre. Une première approche formelle du film, privilégiant les points de contact qu'il entretiendrait avec le néoréalisme, dans la mesure où il s'inscrit en plein dans une saison qui le marque profondément, apparaît comme une façon d'aborder le sens possible de Il Cristo proibito. Cela permettra également d'approfondir la dimension du réalisme particulier de Malaparte et de voir comment ce film s'inscrit dans l'œuvre et les thèmes de l'écrivain, notamment dans les œuvres issues de l'expérience de la guerre dont il fait partie. 


\section{Malaparte et le néoréalisme}

Il peut sembler à première vue tout à fait vain de chercher à repérer les points de contact du film de Malaparte avec un courant - le néoréalisme - qui ne s'est jamais véritablement formalisé ni codifié et qui est déjà lui-même difficilement identifiable. Toutefois, si l'on accepte une certaine vulgate, on peut voir dans le film de Malaparte, ne serait-ce déjà qu'au niveau de son contenu, quelques éléments qui pourraient permettre de l'inscrire dans la mouvance néoréaliste - même s'il reste, comme nous le verrons, fondamentalement étranger à cette esthétique comme à ses présupposés éthiques et idéologiques.

5 Même si l'on sait que les déclarations d'intentions des auteurs sont toujours à prendre avec des pincettes, et celles de Malaparte plus encore qu'aucune autre, on peut partir d'une affirmation du réalisateur lui-même :

Sarà Cristo proibito un film neorealista? Come potrebbe non esserlo?... il mio film vuole essere un omaggio a Rossellini, a De Sica, al Blasetti di Quattro passi fra le nuvole. Ma è naturale che io cerchi di mettere nel film neorealista ciò di cui, a mio parere, esso manca ${ }^{2}$.

6 Par cette affirmation Malaparte range résolument son film à côté des œuvres de réalisateurs qui représentent le néoréalisme le plus classique, le néoréalisme le plus pur (De Sica et Rossellini, notamment ${ }^{3}$ ), tout en indiquant le dépassement que son film entend apporter à leur esthétique. Je voudrais alors essayer de comprendre ce qui "manque », selon Malaparte, aux films néoréalistes et que Il Cristo proibito viendrait ajouter.

7 À un niveau le plus superficiel, celui du contenu, si l'on considère qu'est néoréaliste un film qui s'attache en premier lieu à représenter la dimension historique ou sociale de l'Italie qui lui est contemporaine, Il Cristo proibito s'inscrit sans aucun doute dans cette actualité. Son protagoniste - Bruno - est un soldat qui a fait la guerre sur le front russe, qui a passé plusieurs années dans un camp de prisonniers en Russie avant d'être libéré et qui revient, en 1950, dans son village de Toscane ${ }^{4}$. Malaparte n'invente rien. Il tourne son film au moment où le retour des prisonniers de guerre est une réalité prégnante en Italie et il tient fortement à l'inscription de son film dans cette actualité immédiate :

Deux hommes, deux paysans-ouvriers, reviennent de la guerre, de la captivité. Ils rentrent au village bien longtemps après leurs camarades, car ils reviennent de Russie : leur retour prend place dans le temps, non point en 1945 ou en 1946, mais de nos jours, en 1950 [...] Il fallait qu'ils rentrent de nos jours : et ce n'est que de nos jours que les prisonniers rentrent de Russie ${ }^{5}$.

8 L'inscription de l'histoire du film dans l'actualité contemporaine de l'Italie de l'aprèsguerre en est une dimension essentielle. Tout comme le choix - néoréaliste - de représenter des milieux populaires : les paysans qui en sont les protagonistes souffrent de la misère et du chômage, du manque de terres, de la faim. Le monde de l'aprèsguerre est un monde où règne l'injustice et la polémique sociale est volontairement présente dans le film. L'un des personnages principaux - l'Eremita - professe une sorte de socialisme chrétien et dénonce la société égoïste et capitaliste du début des années 1950. Lors de la procession du jeu de la Croix, le Diable est représenté par un homme à tête de veau qui porte une salopette sur laquelle on voit en gros plan le logo de la marque Pirelli : comme si dans cette société-là, l'ouvrier, le prolétaire, était devenu le Diable, l'ennemi à abattre. Toutefois, la dimension sociale n'est pas, 
fondamentalement, ce qui intéresse le plus Malaparte. Son propos est avant tout moral. Bruno, le protagoniste du film affirme : "Non è la miseria che mi fa paura ». Et, à un disciple de l'Eremita qui lui dit : «Anche il pigliar la terra ai padroni è giustizia », Bruno répond : «Anche questo è giustizia. Ma i nostri soldati sono morti per qualcos'altro ».

9 À cette dimension sociale s'ajoute que le personnage de Bruno - même si Malaparte avait pensé initialement à son ami, l'acteur français Pierre Fresnay, pour tenir le rôle est joué par Raf Vallone qui est en 1950 la star masculine du cinéma néoréaliste, l'acteur fétiche, entre autres, des films de Giuseppe De Santis ${ }^{6}$. Vallone est aussi un acteur engagé politiquement dans les rangs du PCI, journaliste à L'Unità et ancien partisan.

Plus significativement encore, le film de Malaparte s'inscrit dans le contexte de l'aprèsguerre qui est marqué en Italie, et au-delà dans toute l'Europe, par un profond désarroi, par un balancement entre désespoir et espérance, par une profonde crise existentielle et morale liée à la fois aux souvenirs récents de l'horreur de la guerre et à la profonde déception devant l'évolution du monde de l'après-guerre, oublieux des valeurs et des idéaux, notamment ceux de la Résistance, et des sacrifices consentis pour libérer le pays. C'est une période de crise historique mais aussi humaine et philosophique : une crise spirituelle dont Malaparte veut se faire le témoin en cherchant avant tout à montrer les conséquences morales et psychologiques de la catastrophe historique sur ses personnages. Ainsi, ce qui tourmente le personnage, c'est d'abord un climat, comme le proclame la voix off au début du film : «L'angoscia e la speranza del mondo nuovo, del mondo sconosciuto che si apre davanti a loro."

11 Il y a aussi la volonté de Malaparte, proche de celle qui animait les réalisateurs du néoréalisme malgré leurs profondes différences politiques et idéologiques, de faire un cinéma « engagé ». Cela entre dans la conception même que l'écrivain se fait du cinéma qui est pour lui avant tout une arme: "Je sais bien, je suis un blanc-bec du cinéma. Mais quelle importance puisque, selon moi, le cinéma est une arme avant d'être un $\operatorname{art}^{7}$.»

Dans le numéro de Prospettive déjà évoqué, Malaparte, toujours à propos du cinéma, affirme également :

La sua influenza non è unicamente di ordine estetico, ma soprattutto di ordine morale, politico e sociale [...] Il cinema è, prima di tutto, un fatto di cultura, di civiltà. [...] è l'espressione e la rappresentazione del gusto, delle idee, dei sentimenti e dei costumi del proprio tempo, risente del clima storico, politico, sociale, e morale in cui vive .

13 Ce sont justement ces sentiments que Malaparte va chercher à saisir, ceux de ces pauvres gens au sortir de la guerre, et qu'il va chercher surtout à interpréter cinématographiquement. Et selon lui cette interprétation intime ne peut se faire qu'en dépassant la superficialité de la chronique néoréaliste, qu'en la transfigurant esthétiquement ${ }^{9}$ :

Malaparte, a nostro avviso, non resta «dal di fuori» ma entra dentro le cose proprio per non rimanere condizionato dall'esteriorità della cronaca e del documento, credendo (la sola sua unica fede, pensiamo) nell'individuo, nella sua tragedia ${ }^{10}$.

En effet, la plupart des choix opérés par le réalisateur dans son film semblent s'écarter ainsi d'un certain horizon néoréaliste et se situer très nettement en dehors de son esthétique et de son idéologie. Le réalisme de Malaparte n'a rien de naturaliste ni de documentaire, bien au contraire. Son esthétique s'écarte de celle d'un néoréalisme de la 
transparence, du dépouillement - celui d'un Rossellini ou d'un De Sica, par exemple pour se rapprocher en revanche de la tendance baroque du néoréalisme, représentée en particulier par Luchino Visconti mais surtout, encore une fois, par Giuseppe De Santis. En effet, inscrire malgré tout le film de Malaparte dans le courant du néoréalisme, c'est reconnaître l'existence de deux tendances fortes à l'intérieur de ce courant: l'une " ascétique », l'autre « maniériste ${ }^{11}$ » et placer résolument Malaparte à l'intérieur de cette dernière.

De ce point de vue, il ne fait pas de doute que Il Cristo proibito est la mise en œuvre des conceptions du cinéma exprimées par Malaparte en 1951 dans la revue L'occhio magico, en particulier le rejet du documentaire «che di tutti i generi cinematografici è il più retorico, il più aneddotico, il più estetizzante [...] e il più lontano dalla realtà » car « si serve della realtà bell'e fatta, senza neppur tentare d'interpretarla ${ }^{12}$ ».

On trouve dans ces affirmations deux idées particulièrement importantes, que n'aurait pas renié De Santis. En premier lieu, il s'agit d'une violente critique des présupposés qui sont à la base de l'esthétique néoréaliste, à savoir l'importance, la primauté du contenu, l'idée que la réalité n'aurait pas besoin d'être réélaborée, que les faits pourraient parler d'eux-mêmes et révéler ainsi leur vérité. Il y a au contraire chez Malaparte le rejet fort de cette dimension naturaliste, de cette dimension documentaire du cinéma néoréaliste, et l'affirmation de la nécessité d'aller au-delà du simple enregistrement, de l'extériorité de la chronique, du document brut.

La deuxième idée contenue dans la citation nous permet d'expliciter ce qui «manque » selon Malaparte au cinéma néoréaliste. Elle tient dans le verbe "interpréter ». Pour Malaparte, il s'agit bien d'interpréter la réalité, ce que ne fait pas selon lui le néoréalisme qui manque l'essentiel, à savoir "les sentiments", en restant à la surface de la chronique sociale.

Une autre citation de Malaparte sur le cinéma peut continuer à nous aider à mieux élucider les choix expressifs et esthétiques opérés par le réalisateur dans Il Cristo proibito. Elle est tirée à nouveau du numéro spécial de Prospettive de 1937, d'un éditorial fondateur, intitulé Verità e cinema, qui résonne, près de quinze ans avant le tournage de Il Cristo proibito, comme un manifeste programmatique.

Dans cet article, Malaparte considère l'invention du cinéma comme le point d'aboutissement de l'idéal du théâtre romantique, un idéal poursuivi depuis le théâtre élisabéthain, et qui était d'ouvrir au monde lui-même l'espace de la scène :

L'invenzione di Lumière ha permesso al teatro [...] di «liberarsi dalla realtà del teatro» [...] la macchina da presa ha consentito al teatro [...] di capovolgere i suoi rapporti col mondo della natura e della realtà. Nei confronti della natura e della realtà, il teatro rappresentava un artificio, una convenzione. Il cinematografo, al contrario, ha fatto della natura e della realtà un artificio, una convenzione cinematografica. All'antica convenzione: «questa scena rappresenta un bosco» ha sostituito l'altra: «questa scena rappresenta la scena di un bosco». [...] la natura e la realtà come artifici e convenzioni cinematografici, come personaggi e come macchine sceniche ${ }^{13}$.

La réalité elle-même comme artifice et convention cinématographique, comme élément scénique. Il y a là, implicite, l'idée que la réalité peut être transfigurée par la puissance du medium cinématographique, capable de la re-façonner et de s'en servir comme une véritable scène, en soulignant sa dimension artificielle, par saturation des effets naturalistes. 
21 L'image ne chercherait plus alors à donner l'illusion d'une reproduction mimétique de la réalité, comme dans la transparence du néoréalisme classique, mais elle représenterait, remodèlerait cette réalité, chargée - et même surchargée, dans le cas du cinéma de Malaparte - de connotations symboliques, de références religieuses, de citations picturales, de déformations baroques, d'hyperboles exacerbées, d'abstractions expressionnistes visant, entre autres, à sculpter plastiquement les corps et les paysages.

22 Ce véritable théâtre de passions violentes ainsi créé viserait alors bien à « interpréter les sentiments » les plus profonds, les plus primitifs et les plus instinctifs, de ses personnages populaires.

On est là aux antipodes de l'esthétique néoréaliste " ascétique ", mais au plus près de celle, «maniériste » de Visconti, ou d'un De Santis. Je voudrais maintenant le montrer à travers les choix expressifs fondamentaux opérés par le réalisateur dans $\mathrm{Il}$ Cristo proibito.

\section{Une scène de passions primitives}

24 Le premier de ces choix est l'utilisation, dès la séquence d'ouverture du film, de la voix off. Dans cette séquence initiale, qui peut être considérée comme une sorte de prologue, une instance narratrice extradiégétique, une voix, qui est celle d'un ouvrier, met en place une scène («Guardate questo paese. È un pezzo di Toscana») et les protagonistes d'un drame. Il y a déjà là un effet de mise à distance du spectateur, un effet «straniante».

Cette voix off pourrait être aussi qualifiée d'« indigène ». Certes, celui qui parle est un "povero operaio lombardo", mais il connaît par cœur la région où va se dérouler le drame pour y avoir vécu et travaillé de nombreuses années («Conosco questo paese e conosco questa gente »). Il n'est pas indifférent que celui qui sait les choses ne soit qu'un «pauvre ouvrier». C'est ainsi que se définira aussi le personnage de Mastro Antonio : "Non sono che un povero operaio ma certe cose le capisce solo la povera gente. " On a là un dispositif "verghien », pourrait-on dire. La voix off provient de l'intérieur du monde paysan qui va être représenté, elle a fait une expérience que n'a pas encore accomplie Bruno, le protagoniste du film, elle a un savoir qu'il n'a pas encore.

26 Aussi cette instance de narration se montre-t-elle d'emblée ironique et sarcastique visà-vis du protagoniste du film, établissant ainsi une distance entre ce qu'elle sait, elle, et ce qu'ignore encore Bruno, le personnage, et dont il va faire l'expérience tout au long de la narration filmique: "Poveretto! Crede ancora nella giustizia che uno si fa con le proprie mani. E non sa che sono sempre gli innocenti a pagare per gli altri. » Ce ton du sarcasme renforce la dimension « verghienne » que nous évoquions dans la mesure où le « sempre » renvoie à une vision fataliste et déterministe, "victimisante ", de l'histoire et des rapports sociaux, ce que l'épilogue du film viendra confirmer.

En tout cas, ce dispositif initial singulier implique trois choses.

Tout d'abord, il construit la dimension littéraire d'un film qui est - comme je l'ai dit adapté d'un roman: l'insertion dans le film de ce commentaire en voix off extradiégétique constitue une trace de l'état antérieur de l'œuvre. Il sert de porte-parole à l'auteur lui-même et porte d'emblée des jugements dont le personnage principal va 
ensuite éprouver la validité. C'est un commentaire qui semble mettre en place un récit de type scriptural plutôt qu'un récit de type mimétique.

En second lieu, la présence de cette voix qui sait déjà tout construit la dimension fortement démonstrative du film, dont on peut dire qu'il s'agit clairement d'un « film à thèse ». Renforcée par le plan-séquence aérien qui surplombe et englobe les personnages, le paysage et toute l'histoire, comme pour signifier la souveraineté écrasante du destin dominant les simples mortels, l'injonction faite par la voix off au spectateur («Guardate ») place celui-ci dans la position d'un observateur frontal de ce monde (mais qui est aussi le sien, puisque le film s'adresse au public populaire italien), comme si allait avoir lieu, devant lui, la représentation d'une histoire exemplaire, édifiante et même éducative.

Enfin, ce dispositif souligne le rapport entre la voix off et le personnage. Ici, au début du film, ce rapport est marqué par une grande distance, mais celle-ci va se réduire jusqu'à disparaitre à la fin du film, dans ce qui peut être considéré comme un épilogue (sur lequel nous reviendrons) où l'on retrouvera en partie le même dispositif, à ceci près que les hurlements du personnage et les invectives de la voix off tendront à se confondre et à ne faire plus qu'un, jusqu'à ce qu'il soit impossible de savoir qui parle. À ce moment-là, le protagoniste aura enfin compris, parce qu'il en aura fait lui-même l'expérience, la triste vérité morale que savait dès le départ la voix off.

De ce point de vue, le film est aussi un film circulaire. Dans l'encadrement épilogue/ prologue va se mettre en place la démonstration d'un échec. Là encore, on peut reconnaître la marque d'un réalisme de type "verghien ». L'histoire représentée à l'intérieur du cadre, qui en fait comme une parabole exemplaire, va laisser apparaître la présence d'un fatum, inexorable et implacable, guidé par des forces inflexibles. Il n'y aura pas à la fin du film rupture du cercle mais au contraire continuité d'un cycle pérenne : celui de la défaite et du sacrifice des innocents.

Un autre procédé récurrent qu'utilise Malaparte est celui de la symbolisation. Dans la plupart des plans, le fond et le décor (les murs couverts d'inscriptions funéraires, les crucifix, les tableaux, les photos, les affiches, les inscriptions, etc.) connotent symboliquement le sens de l'action et l'univers moral des protagonistes. Malaparte travaille le plan dans une perspective de symbolisation permanente, à commencer par celle du paysage lui-même, qui l'éloigne fortement de la tendance mimétique et documentaire du néoréalisme.

Pour revenir à la séquence initiale du film, les deux protagonistes parcourent un paysage de collines aride et désert, aussi pelé qu'un calvaire, parsemé de dizaines de tombes surmontées de petites croix blanches. Un paysage presque halluciné où la mort est partout. La guerre - cette «bella porcheria » - n'est plus envisagée ici seulement dans sa dimension historique mais aussi comme le symbole d'un enfer humain, d'une malédiction universelle, d'un monde entaché d'une faute collective. Elle y devient la marque d'une humanité maudite.

34 Il y a tout au long du film d'autres plans fortement, voire outrancièrement, saturés de symboles, le plus souvent religieux. Par exemple, le gros plan sur la tête de veau dans la boutique du boucher puis, plus tard, le même animal que le même boucher « mussolinien » met à mort, comme un sacrifice : « image de l'innocence souillée par les hommes et par leur folle descente dans la peste de l'Histoire", image d'« animaux innocents qui portent en silence le poids de la faute des hommes ${ }^{14} »$. À travers cette forte connotation symbolique, la figure de l'agneau massacré assume la fonction de la 
victime sacrificielle et évoque bien sûr le sacrifice de l'agneau divin. Il est aussi la métaphore d'une condition humaine dominée par la soif de vengeance : il anticipe ce qui pourrait arriver au meurtrier du frère du protagoniste si ce dernier ne renonçait pas à accomplir jusqu'au bout son désir de vengeance ${ }^{15}$. Il symbolise enfin le sacrifice des innocents qui est le leitmotiv idéologique du film.

Cette figuration symbolique de l'innocence se retrouve aussi à travers le montage dans l'utilisation de collisions signifiantes et expressionnistes, suivant la leçon d'Eisenstein, lorsque Malaparte, dans un plan diégétiquement inutile, oppose la pureté et l'innocence d'une mère qui tient un enfant dans ses bras et repousse du pied un couteau, au boucher qui immole un animal et à la foule en transe qui accourt au spectacle d'une vengeance qu'elle craint imminente.

Dans l'épilogue, l'habitation de Mastro Antonio, où ce dernier vient de se sacrifier, se dresse comme une sorte de croix érigée sur un calvaire avec, dans le fond, un ciel tempétueux d'apocalypse ${ }^{16}$. Tout au long du film, les parallélismes entre la mère de Bruno et la Vierge sont nombreux : "Domani è la festa della Madonna, ma stasera è la tua festa, mamma.» Quant à Bruno, il ne veut rien savoir de sa mère («non voglio sapere nulla da te mamma ») afin qu'elle reste pure, immaculée, et qu'elle ne soit pas à l'origine de la vengeance. Pourtant, c'est elle qui va trahir et se trahir : à ce moment-là, Bruno la repoussera violemment.

Mais il y a une scène en particulier, sans doute la plus belle du film, qui nous permet d'approfondir l'utilisation de ce procédé de la symbolisation ainsi que celui des récurrentes citations picturales disséminées à l'intérieur de nombreux plans. Cette scène, c'est celle de la mort de Mastro Antonio.

38 Le charpentier Antonio meurt crucifié. Non pas exactement comme le Christ (Malaparte s'en est défendu) mais comme un homme-Christ, un homme qui répète, qui rejoue, qui re-présente le sacrifice du Christ pour sauver les autres hommes. La profondeur de champ du plan où l'on voit Antonio étendu sur son établi fait de ce dernier un gisant. L'image est évidemment inspirée de La lamentation sur le Christ mort de Mantegna ${ }^{17}$.

39 Ce procédé de "picturalisation » du plan a différentes visées. Il est sans doute en grande partie l'œuvre du décorateur du film, le peintre Orfeo Tamburi, ami de Malaparte. Il est important d'en faire quelques autres exemples pour continuer à approfondir les dimensions du réalisme singulier de Malaparte.

40 Il est présent dès la séquence initiale, déjà évoquée, à propos de laquelle Malaparte a dit lui-même qu'il avait voulu retrouver les paysages primordiaux, durs et arides, l'univers archaïque de Giotto, de Masaccio et de Piero della Francesca :

Per gli esterni ho scelto una regione d'Italia dove la natura è magra, severa, essenziale, nuda. L'Italia di Giotto, di Masaccio, di Piero della Francesca: povera d'alberi, di acque, nuda di messi, una distesa ondeggiante di crete dove vive un popolo magro, taciturno, pieno di una profonda vita morale. La civiltà italiana è nata lì, in quelle terre, e quello è il popolo più antico d'Italia ${ }^{18}$.

41 Ces références à la peinture de Giotto ou de Piero della Francesca, les maitres italiens de la perspective, visent à styliser géométriquement les plans. On retrouve cet ordre net des lignes, verticales et horizontales, dans la représentation des intérieurs des habitations paysannes. Cette première forme de stylisation et d'abstraction du paysage et des intérieurs est là pour signifier que ce qui est en jeu n'est pas seulement l'actualité, la chronique sociale, mais aussi la représentation d'une Toscane primitive 
et, au-delà, d'une civilisation immémoriale - «uno dei paesi più antichi e civili dell'Italia e del mondo » comme le désigne la voix off - détruite par le conflit mondial et par une lutte fratricide qui elle-même prend alors, dans ce cadre abstrait et géométrique, des allures de drame sacré.

Dans cette même perspective, le conflit moral et interpersonnel qui habite chacun des personnages du film met en jeu lui aussi des passions primordiales et des pulsions élémentaires : il est dramatisé par les éclairages tranchants et expressionnistes d'un noir et blanc glacé que l'on doit à l'opérateur du film, Gabor Pogany. Ce dernier fait un usage massif du clair-obscur, des jeux expressionnistes de l'ombre et de la lumière, afin d'exacerber les conflits et souligner la double nature des personnages, à la fois innocents et souillés.

De ce point de vue-là, la longue séquence du jeu de la Croix est significative. Les plans d'ensemble fixes en plongée sur la foire populaire qui accompagne la procession installée sur la place de Montepulciano font penser au célèbre tableau L'Entrée du Christ à Bruxelles du peintre belge James Ensor. De manière plus générale, les representations expressionnistes de toute la séquence du jeu de la Croix ainsi que la thématique socioreligieuse qui la traverse rappellent les orientations esthétiques et idéologiques du peintre d'Ostende: reprise de la tradition du masque, dimension grotesque et carnavalesque, dramatisation des effets de lumière, ironie grinçante, alliance de l'étrange et de la critique sociale. Parallèlement, elles rappellent aussi, avec la reprise de la rhétorique de la fête populaire et de ses connotations carnavalesques, de la figure de la place, du fourmillement de la foule minuscule, prise de loin et en plongée, les scènes villageoises (La danse de la mariée en plein air par exemple) à la fois réalistes et grinçantes de Brueghel l'Ancien.

Ce formalisme éloigne en tout cas les plans du film d'une représentation mimétique de la réalité. Au contraire, ils la ré-élabore en permanence, selon les conceptions du cinéma exprimées par Malaparte. Maniérisme baroque, expressionnisme violent, symbolisation permanente et jeu de la citation picturale caractérisent ce réalisme qui vise non pas la transparence de la chronique sociale d'un De Sica, mais, sur le versant d'un De Santis, à produire la synthèse presque "mythique » d'une société, qui entend non pas décrire, rester à la surface, mais interpréter les passions primordiales des protagonistes, c'est-à-dire, à travers ces procédés formels qui conduisent à la stylisation du paysage, des intérieurs et des personnages mis en scène, à toucher le cœur des pulsions élémentaires qui les animent.

Dans cette perspective, le procédé le plus prégnant est sans aucun doute celui de la théâtralisation de la réalité.

Il s'inscrit tout d'abord au niveau de la structure narrative du film. Malaparte a déclaré à plusieurs reprises que son écriture littéraire était déjà, en elle-même, cinématographique puisqu'il écrivait par blocs, par séquences ${ }^{19}$. De la même manière, Il Cristo proibito est un film loin de la chronologie linéaire et de la dimension narrative. Il se découpe en réalité en quatre grands tableaux, en quatre grands actes où se multiplient les scènes de confrontation entre les personnages : une scène d'exposition durant laquelle Bruno se confronte aux habitants du village, deux scènes qui mettent en scène des rituels, des fêtes à la fois païennes et religieuses (le jeu de la Croix et la fête des vendanges, pendant laquelle a lieu la longue scène de la confrontation entre Bruno et Antonio), enfin le dénouement du drame (la confrontation entre Bruno et le traitre Pinin) suivi d'un épilogue. Tout cela a lieu sur trois jours. 
47 Au niveau de la thématique du film, on repère la présence de la fatalité, du fatum tragique. Comme dans la tragédie grecque, la Justice est le sujet central du film. La fatalité qui pèse sur Bruno, c'est le désir de vengeance et de justice individuelle : « Sono tornato solo per questo: per sapere chi è stato.» Ce désir fait de lui « un angelo della vendetta ${ }^{20} »$.

L'autre thème du film - tragique, lui aussi - est celui du retour. Le retour d'un survivant que l'on croyait mort et qui revient pour se venger. La guerre habite encore Bruno et il cherche à la perpétuer en tuant l'assassin de son frère. À ce propos, Luigi Martellini évoque le parallèle avec le protagoniste de Mamma marcia qui affirme :

Per un uomo che ha fatto la guerra, tutta la sua vita non è che uno scuro, profondo, inconscio ricordo della guerra, e dei suoi orrori [...] non essendo l'uomo riuscito a dimenticare i fatti atroci nascosti dentro la memoria [...] dopo il ritorno, quell'uomo continua a stare in guerra, resta con l'idea fissa dell'uccisione ${ }^{21}$.

De la même manière, dans le film, Bruno confesse : « Ho l'inferno dentro di me.»

La guerre - «quella cosa sporca », "quegli anni maledetti»- a fait tomber sur lui cette malédiction et l'a transformé, comme l'indique, dès la séquence initiale, le moment où Bruno regarde son propre visage dans l'eau et crache dessus ${ }^{22}$. Mastro Antonio le lui dit aussi: "Lasciati guardare Bruno. Come sei cambiato. Quando sei partito eri un uomo fatto ma avevi ancora gli occhi di un ragazzo. » C'est que la guerre - cette autre figure de la fatalité - a fait perdre l'innocence et a souillé le monde. Tous les personnages ont été salis par elle, tous ont perdu leur innocence, comme Nella le dit à Bruno : « È venuta la guerra e ha sciupato tutto [...] Non siamo più quelli di una volta [...] Non puoi sapere quello che la guerra ha fatto di noi. » Tous les habitants du village, à la fois innocents et coupables, à la fois victimes et bourreaux, sont porteurs d'une souffrance mais aussi d'une faute, d'une haine refoulée en eux : Maria a trahi Bruno et s'est donnée à Giulio ; Nella s'est vendue aux Allemands («Ho fatto la puttana per salvare gli altri »); le père de Bruno ne pardonnera jamais; sa mère refoule sa haine jusqu'à son lapsus révélateur; Antonio est un assassin, Pinin un traitre. Plus personne n'est innocent, sauf peut-être les morts ${ }^{23}$.

Tous les personnages sont aussi, comme dans une tragédie, liés entre eux et liés par la faute : Nella, l'amoureuse de Bruno avant la guerre, est la sœur du traitre Pinin ; Maria, la sœur adoptive de Bruno, l'a trahie avec son frère. L'amour d'avant la guerre est désormais impossible entre Bruno et Maria ou entre Bruno et Nella: les deux scènes d'amour, très chastes, entre ces personnages soulignent l'impossibilité d'un baiser parce qu'il n'y a plus d'innocence, littéralement et symboliquement : « Tu non puoi più volermi bene. Io non sono più come mi hai lasciata ", dit Maria à Bruno.

Bruno est donc un personnage agi par son destin qu'est son désir de vengeance. Mais au-delà de Bruno, au-delà de cette situation historique particulière qu'il incarne, sa malédiction est aussi, comme dans le dispositif tragique, celle de l'Homme en général, celle d'une Humanité qui croit en la vengeance comme à une possible rédemption, à une possible libération du Mal, alors qu'elle ne fait que le perpétuer. En le perpétuant, elle n'en finit pas, comme la guerre, de tuer l'Humanité : «Un uomo che ammazza un altro ammazza sempre se stesso ${ }^{24}$.» Ou encore, comme le dit Antonio à Bruno : «Un assassino, ecco quello che sarai per tutta la vita se io non riuscirò a salvarti. » L'enjeu du drame est alors de savoir s'il est possible de se libérer, de retrouver l'innocence pour sortir du cycle infernal de cette violence, d'empêcher que « le bourreau ne survive dans la soif de vengeance qui anime la victime ${ }^{25} »$. 

d'abstraction qui vise à faire des différents personnages de purs symboles. Ces derniers prennent la dimension d'entités primordiales, la fixité d'idoles primitives, un caractère presque totémique. Ils incarnent en réalité des positions philosophiques et existentielles. En surjouant leur attitude, en travaillant sur l'exaspération des postures, ils se monumentalisent, à travers un jeu souvent expressionniste et straniato, toujours hyperbolique, entre la fiction et son dévoilement. Comme le souligne Maurizio Serra, ce « côté statique de la prise de vues [...] doit communiquer le sens de la fatalité qui pèse sur les hommes ${ }^{28} »$. Les actions des personnages sont alors le déroulement, déjà inévitablement sanctionné dès le prologue du film, d'un destin. À la fin, malgré la tentative d'y échapper, la Loi tragique qui condamne les innocents ne sera pas brisée. La parabole ne finit pas sur une intervention populaire aux pouvoirs thaumaturgiques qui viendrait lever la malédiction qui pèse sur les innocents sacrifiés. Malaparte n'y croit pas.

dimension théâtrale et statuaire ${ }^{29}$. Cette littérarité, totalement anti-naturelle, est aux antipodes de l'esthétique du dialogue néoréaliste. La spontanéité, le mimétisme de la langue verbale, l'usage du dialecte, sont totalement absents du film.

57 Le réalisme de Malaparte est donc bien l'antithèse du néoréalisme de la transparence dans la mesure où il se construit par exaspérations à tous les niveaux de la mise en 
scène. Il restitue certes le monde populaire paysan mais sous la forme d'une tragédie archaïque, où l'action et les personnages, bien qu'ancrés dans une actualité historique, prennent la forme de figures essentielles, mues par des pulsions primitives (la soif de vengeance, la trahison, le sacrifice, le sexe, la solidarité, la passion), dans un univers rendu abstrait par la mise en scène, où les éléments naturels du paysage se transforment à leur tour en symboles. D'une certaine manière, Malaparte cherche à synthétiser sous une forme primordiale, "mythique ", à placer dans une sorte d'atemporalité et sur une scène tragique, une histoire qui s'inscrit au départ dans une réalité historique et sociale et à lui donner par là la force morale et édifiante d'une parabole sur l'Humanité.

\section{Hypothèse(s) sur le sens de la parabole}

Après avoir tenté d'en analyser la forme, je voudrais m'arrêter désormais sur les pôles mêmes de cette parabole tragique, sur la fonction de ceux qui en sont ses principaux protagonistes, même s'il est toujours malaisé, avec Malaparte, de dégager un sens univoque du discours.

On pourrait pour cela commencer par une expression du réalisateur lui-même qui indique que le drame dont il est question dans son film est «le drame de l'innocence ». Nous l'avons dit: la guerre a souillé le monde, l'innocence a été perdue. Dans ces conditions, un retour à l'innocence, qui laverait le monde du sang et des horreurs de la guerre, est-il possible, sans pour autant qu'à nouveau des innocents le payent de leur vie? Une sortie de ce cycle de répétition tragique de la violence, dont les innocents sont les victimes désignées, est-elle envisageable?

Dès le début du film, la voix off pose les données du drame dans la confrontation qu'elle établit entre Bruno, assoiffé de vengeance, habité encore par la violence de la guerre, prêt à continuer à faire couler le sang, et les autres villageois, qui rejettent cette violence et redoutent le retour aux luttes fratricides.

61 Toutefois, les habitants du village, métaphore d'une Humanité issue de la guerre, rejettent le sang versé par peur et par égoïsme, par crainte de voir resurgir la violence passée. En cela, ils aliènent et s'aliènent, dans le monde matérialiste et égoïste de l'après-guerre, la vraie liberté pour laquelle des milliers d'innocents sont morts et qui se sont sacrifiés, peut-être pour rien : « Neppure la libertà ha riuscito a fare di noi degli uomini liberi », affirme Bruno. En errant dans les rues désertes et silencieuses du village, en pleine nuit, en apostrophant du regard les autres villageois, avec un regard chargé de reproches, en dévoilant le sentiment de culpabilité de tous, il est comme un spectre vengeur, «lo spettro di quella libertà e di quella giustizia per cui tutti hanno sofferto ${ }^{30} »$.

62 L'un des protagonistes du drame est donc le village, symbole d'une Humanité qui ne sait plus souffrir pour les autres, qui ne croit plus, au sortir de la guerre et dans le monde décevant de l'après-guerre, en la libération à travers le sacrifice pour autrui, qui fut la leçon laissée aux hommes par le Christ. Cette incapacité fait que les hommes, pourtant objectivement libérés par le sacrifice de milliers d'innocents, se retrouvent aussi peu libres qu'avant, enchaînés qu'ils sont à leur propre souffrance, à leur propre égoïsme et à leur propre résignation. Là peut se trouver le sens du titre du film - Il Cristo proibito - dans ce constat amer que fait Malaparte : dans la société moderne, si peu spirituelle et outrageusement individualiste, capitaliste et matérialiste, il est 
interdit - interdiction à la fois imposée à et intériorisée par chacun - de se comporter comme un Christ, de renouveler le sacrifice du Christ, c'est-à-dire d'aimer et de souffrir pour les autres. C'est en ces termes que le personnage de l'Eremita, défenseur d'une sorte de « socialisme chrétien » en fait le reproche à ses semblables :

Finché tu soffri per la tua fame, per la miseria tua, della tua donna e dei tuoi figli, finché ti avvilisci e ti rassegni, allora tutto va bene. Ma appena tu soffri per la fame degli altri, per la miseria dei figli degli altri, per l'umiliazione degli altri uomini, allora sei un uomo pericoloso, un nemico della società.

Mais l'Eremita reste dans une dimension politique et sociale. Peut-être que la dimension la plus profonde de la parabole est celle, plus spirituelle, qui se joue entre le pardon et la vengeance, ces "deux polarités qui ne peuvent se réconcilier ${ }^{31}$ » chez Malaparte, entre le rachat et la malédiction, entre le sang versé en sacrifice, pour les autres, et le sang qui tue, entre le geste du Christ et celui de Cain ${ }^{32}$. Cette série d'oppositions s'articule principalement et symboliquement autour de la confrontation entre deux personnages : Bruno et Mastro Antonio. «Solo il sangue che un uomo versa per gli altri uomini è una cosa pura [...] Non c'è giustizia in chi uccide [...] Solo nel sacrificio è giustizia » affirme ce dernier, qui défend la force du pardon, de l'amour de l'autre et du sacrifice.

Mastro Antonio apparaît ainsi dans le film comme le personnage porte-parole des conceptions de Malaparte lui-même. Se définissant lui aussi comme un "povero operaio ", il reprend le discours initial de la voix off, notamment dans la scène capitale de sa confrontation avec Bruno. Selon Mastro Antonio seul le sacrifice qu'accomplit un homme, par amour pour les autres hommes, sur le modèle de celui du Christ, peut sauver l'Humanité. Mais il est important de souligner encore une fois que Malaparte s'est défendu d'avoir voulu faire de Mastro Antonio le symbole du Christ $^{33}$. Car le sacrifice du Christ n'a pas eu lieu une fois pour toutes. Au contraire, il doit être sans cesse renouvelé par chacun d'entre nous. Mastro Antonio reste un homme, et même un homme coupable, mais qui peut dire : «Ho tutti i vostri peccati e i vostri delitti sulla coscienza e sono pronto a pagare per voi. » Chaque homme peut - et doit - être en mesure de renouveler le sacrifice du Christ. Chaque homme peut être un Christ, un Christ pour tous les autres hommes, et être en mesure, par son amour et son sacrifice pour autrui, de sauver l'Humanité, d'en laver les fautes ${ }^{34}$. C'est en cela que consiste la responsabilité morale individuelle de chaque homme, son problème de conscience, dans ce qui pourrait ressembler à une sorte de " communion des saints", en dehors de toute religion institutionnalisée : «ll popolo del Cristo è infatti un popolo libero, e civile, che da se solo sa trovare le vie della propria salvezza, così nel campo sociale come in quello morale ${ }^{35}$."

Le sacrifice, tel qu'il est défini par Mastro Antonio, redonnerait alors un sens à celui des milliers de morts innocents de la guerre. En revanche, la vengeance à laquelle Bruno s'apprête à s'abandonner ne fera que perpétuer le cycle de la violence, de la haine et du sang, comme si les horreurs de la guerre n'avaient servi à rien : « Lo hai già negli occhi il delitto e quella cosa sporca che ha negli occhi tu la chiami giustizia.» Ainsi, pour tenter de sauver Bruno, de l'arracher à son fatum de violence, Mastro Antonio se sacrifie. Il donne sa vie pour sauver à la fois Bruno et celui qui doit être sa future victime, Pinin, que Bruno épargnera, justement en se souvenant du sacrifice de Mastro Antonio : « Ha pagato anche per lui. » 

Pinin, l'horreur de la guerre) et arrêté la fatalité de la vengeance future à laquelle Bruno s'apprêtait à succomber. Par son sacrifice, il semble sauver symboliquement l'Humanité tout entière: "Se un uomo si offrisse di morire per gli altri gli uomini sarebbero liberati dall'odio e dalla violenza. » Toutefois, si dans une première version le film se finissait sur cette note optimiste, sur la possibilité de laver le sang par le sacrifice, les différentes corrections qu'y a apportées Malaparte rendent sa fin beaucoup plus problématique et viennent au contraire sanctionner la vision pessimiste d'un échec.

67 Certes, une fois l'heure de la vengeance écartée, le village reprend vie au son des cloches qui se mettent à carillonner et qui semblent tout d'un coup redonner vie à tous les personnages, recomposer un tissu social harmonieux à l'intérieur duquel Bruno retrouve sa place, comme si le sacrifice de Mastro Antonio avait sauvé la communauté et ressuscité la solidarité et la fraternité humaines. Pourtant, ce n'est pas là le final du film. Il y a, après cette séquence, deux autres séquences : celle de l'ultime confrontation de Bruno avec sa mère, puis celle de Bruno gravissant le calvaire au sommet duquel se tient, droite, la tour-croix de Mastro Antonio.

Or, ces deux séquences établissent un constat d'échec sanctionné d'abord par la réplique de Bruno lors de l'aveu de son crime à sa mère ( Non volevo che un innocente pagasse per gli altri »), puis par le refus qu'il oppose aux larmes et au pardon de cette dernière : «Malaparte n'arrive pas jusqu'au bout de la démonstration, il ne parvient pas jusqu'à croire au message positif qu'il veut communiquer ${ }^{36}$. »

69 Le cours du destin ne s'inverse pas, malgré le geste sacrificiel de Mastro Antonio, et même, au contraire, il ne s'inverse pas à cause de ce geste qui a fait paradoxalement s'accomplir jusqu'au bout non seulement la destinée individuelle de Bruno - se venger en tuant un innocent - mais aussi, à travers lui et son geste, se répéter le cycle tragique dans lequel est prise l'Humanité tout entière qui toujours fait verser le sang d'un innocent pour payer pour les fautes des coupables et des pécheurs.

Dans la dernière séquence du film, pour expier sa propre faute, Bruno doit alors gravir à son tour, comme l'avait fait avant lui Mastro Antonio, son calvaire. C'est là qu'il comprend et qu'il se met à hurler contre ce commun et injuste destin humain ${ }^{37}$. Le ton des « Perché ", déchirants, répétés anaphoriquement pas moins de treize fois, n'est plus celui de l'interrogation, de l'ignorance du Bruno de la séquence initiale, mais bien celui de l'invective d'un personnage qui sait désormais le tragique de sa condition.

71 La parabole mise en scène par Malaparte n'ouvre pas sur ce qui aurait pu être une fable utopique, destinée, de manière édifiante, à montrer la voie vers la construction d'un monde idéal et meilleur, où régnerait, enfin, la justice. Il n'y a pas, à la fin, de sortie du cycle tragique de la répétition de la violence et du sacrifice des innocents. Il n'y a pas de rédemption possible pour l'Humanité, ni de retour à l'innocence après l'horreur de la guerre : «Le Christ demeure interdit, à la fin comme au début du film ${ }^{38}$. »

La fin est une fin désespérée au sein d'un prologue qui rappelle l'épilogue et souligne la circularité de l'histoire et de l'Histoire humaine. On y retrouve la voix off mais dans un dispositif quelque peu différent : on ne sait plus si c'est le personnage qui hurle ou bien la voix off, voire le réalisateur lui-même. Plus vraisemblablement, il s'agit des trois ensemble, comme si l'expérience traversée par le personnage lui avait fait comprendre

Cahiers d'études italiennes, 24 | 2017 
la leçon et reprendre à son compte le constat désespéré du début du film: dans le monde, ce sont toujours les innocents qui paient.

\section{NOTES}

1. Pour des informations très complètes sur la genèse du film, voir L. Martellini, « Malaparte fra letteratura e cinema», Chroniques italiennes, n 44, 1995/4 (numéro épuisé). L'article est consultable en format pdf à partir du lien <http://chroniquesitaliennes.univ-paris3.fr/PDF/44/ Martellini.pdf>. C'est à cet article en ligne que nous ferons référence. Voir également la monographie très précieuse sur le film, qui contient les différentes versions du scénario et les dialogues du film, L. Martellini, Il Cristo proibito, Naples, Ed. Scientifiche italiane, 1992.

2. G. P. Brunetta, Storia del cinema italiano. Dal neorealismo al miracolo economico. 1945-1959, Rome, Editori Riuniti, 1998, p. 517.

3. Paradoxalement - et je m'attacherai à le montrer - si le film de Malaparte est susceptible d'entretenir des liens esthétiques et stylistiques avec le néoréalisme, ce n'est certainement pas avec ces deux réalisateurs-là !

4. Au niveau de la ligne générale de la diégèse, on remarque une première ressemblance avec le film de Giuseppe De Santis intitulé Non c'è pace fra gli ulivi (1950), film avec lequel Il Cristo proibito entretient plus d'une similitude. Nous y reviendrons.

5. Lettre de Malaparte à son ami l'acteur français Pierre Fresnay, citée par M. Serra, Malaparte, vies et légendes, Paris, Tempus Perrin, 2012, p. 523.

6. Raf Vallone est au même moment le héros positif de deux des trois premiers films de Giuseppe De Santis - Riso amaro (1949) et Non c'è pace fra gli ulivi (1950) - qui constituent, avec Caccia tragica (1947), la fameuse «trilogie de la terre».

7. C. Malaparte, Carrefour, 12 juin 1951, cité par L. Martellini, Il Cristo proibito, ouvr. cité, p. 58.

8. C. Malaparte, «Verità sul cinema ", Prospettive, 1937, cité par L. Martellini, "Malaparte fra letteratura e cinema ", ouvr. cité, p. 7. Nous soulignons.

9. C'est là encore un point qui rapproche Malaparte et De Santis.

10. L. Martellini, « Malaparte fra letteratura e cinema », art. cité, p. 3.

11. S. Parigi, Neorealismo. Il nuovo cinema del dopoguerra, Venise, Marsilio, 2014, p. 210.

12. C. Malaparte, «Regista compositore e regista direttore", L'occhio magico, 1951, cité par L. Martellini, « Malaparte fra letteratura e cinema », art. cité, p. 5.

13. C. Malaparte, «Verità sul cinema », Prospettive, 1937, cité par L. Martellini, Il Cristo proibito, ouvr. cité, p. 57-58.

14. M. Serra, Malaparte, vies et légendes, ouvr. cité, p. 524-525.

15. Il anticipe avant tout le sacrifice de Mastro Antonio qui, innocent, décide néanmoins de payer pour Pinin et de rejouer le sacrifice du Christ pour le salut du monde.

16. L. Martellini, Il Cristo proibito, ouvr. cité, p. 41.

17. Même si P. P. Pasolini n'a jamais fait mention d'avoir vu un jour le film de Malaparte, ce plan semble anticiper, de manière troublante, le plan quasi identique d'Ettore mort dans Mamma Roma (1962). Pour les nombreuses similitudes entre Il Cristo proibito et les films de Pasolini, notamment Accattone (1961), Mamma Roma (1962) et Teorema (1968), voir L. Martellini, Il Cristo proibito, ouvr. cité, p. 52.

18. Ibid., p. 31. 
19. Voir C. Malaparte, «Appunti per un'intervista », dans M. A. Prolo, Curzio Malaparte e il cinema, Turin, Museo Nazionale del Cinema, 1967, cité par L. Martellini, Il Cristo proibito, ouvr. cité, p. 27-28.

20. Ibid., p. 38 .

21. Ibid., p. 31.

22. Dans une autre séquence du film, Bruno observe son portrait fracturé dans un miroir et Maria lui ordonne : « Retourne ce miroir. »

23. Là aussi Malaparte prend le contrepied, comme De Santis, de la vision néoréaliste paternaliste, sentimentale, et pour finir mystificatrice, d'un peuple par nature bon et sain. Dès la première séquence, à Andrea qui lui dit, en parlant de la guerre et de ses malheurs, « Non è colpa nostra ", un paysan sur sa mule répond : « È colpa nostra, è colpa di tutti »; et Bruno renchérit : «Ha ragione lui. È anche colpa nostra. È colpa di tutti. » La cruauté, le désir de tuer, la soif de vengeance, toutes ces passions élémentaires et brutales, font partie chez Malaparte du monde intérieur de ses personnages populaires.

24. Cité par L. Martellini, Il Cristo proibito, ouvr. cité, p. 51.

25. M. Serra, Malaparte, vies et légendes, ouvr. cité, p. 527.

26. Gianni Rondolino souligne à juste titre qu'avec son film Malaparte retrouve « $\mathrm{i}$ toni e timbri della tragedia antica, pur calando fatti e personaggi nell'ambiente contadino di un'Italia sconvolta dalla guerra, con le ferite non ancora rimarginate ». Le critique rapproche aussi, comme nous l'avons fait déjà fait à plusieurs reprises, le style de Malaparte de l'esthétique de Giuseppe De Santis en affirmant qu'avec Il Cristo proibito, Malaparte « indicò una possibile strada per superare i limiti del neorealismo [...] richiamandosi per certi aspetti al cinema di Giuseppe De Santis » (G. Rondolino, Il neorealismo italiano, dans Storia del cinema, vol. II, Turin, UTET, 1977, p. 397-399 et 481-482).

27. L. Martellini, « Malaparte fra letteratura e cinema », art. cité, p. 8-9.

28. M. Serra, Malaparte, vies et légendes, ouvr. cité, p. 526.

29. On peut y voir, moins positivement, avec l'historien du cinéma Gian Piero Brunetta, qui éreinte violemment le film, l'un de ses (nombreux) défauts : « Il testo verbale non scende mai dal suo registro letterario [...] non riesce a far corpo con l'immagine.» (G. P. Brunetta, Storia del cinema italiano. Dal neorealismo al miracolo economico. 1945-1959, ouvr. cité, p. 518.)

30. L. Martellini, Il Cristo proibito, ouvr. cité, p. 35.

31. M. Serra, Malaparte, vies et légendes, ouvr. cité, p. 525.

32. "Solo la morte di un innocente placherà gli odi di una umanità in cui il "fratello" uccide il "fratello", rinnovando ogni giorno il gesto di Caino. » (L. Martellini, Il Cristo proibito, ouvr. cité, p. 50.)

33. Même si toute la séquence de la confrontation finale entre Bruno et Mastro Antonio est saturée de symboles christiques : l'établi du charpentier, la « crucifixion » de Mastro Antonio, les allusions visuelles à une déposition de la Croix lorsque Bruno recueille dans ses bras la dépouille de son ami, la citation, enfin, du tableau de Mantegna, déjà évoquée.

34. On peut renvoyer, avec Luigi Martellini, à la fin de La pelle et à la conversation entre Malaparte et son ami Jimmy Wren : "Lo sai anche tu che non è vero che Cristo ha salvato il mondo una volta per sempre. Cristo è morto per insegnarci che ognuno di noi può diventar Cristo, che ogni uomo può salvare il mondo col proprio sacrificio. Anche Cristo sarebbe morto inutilmente, se ogni uomo non potesse diventar Cristo e salvare il mondo.» (C. Malaparte, La pelle, Milan, Mondadori, « Gli Oscar », 1991, p. 324-325.)

35. Extrait du communiqué de presse du film, dactylographié et non publié, cité par L. Martellini, Il Cristo proibito, ouvr. cité, p. 44.

36. M. Serra, Malaparte, vies et légendes, ouvr. cité, p. 527. On a là une grande différence avec Non c’è pace fra gli ulivi, le film de Giuseppe De Santis que nous avons évoqué à plusieurs reprises, qui 
se termine positivement et indique la voie vers la construction idéale d'un monde populaire où régneraient les valeurs de fraternité et de justice.

37. L. Martellini, Il Cristo proibito, ouvr. cité, p. 41.

38. M. Serra, Malaparte, vies et légendes, ouvr. cité, p. 527.

\section{RÉSUMÉS}

Il Cristo proibito est le seul film tourné par Curzio Malaparte. Sorti en 1951, il s'inscrit dans la période historique de l'après-guerre et doit se positionner par rapport au courant esthétique dominant de l'époque : le néoréalisme. À travers une analyse approfondie des séquences du film et la relecture des textes théoriques de Malaparte sur le cinéma, cet article essaie de replacer le film dans ce contexte afin d'éclairer l'approche malapartienne originale de la réalisation cinématographique et de remettre cette œuvre maniériste et ambiguë au cœur des thématiques et des obsessions de son auteur.

Il Cristo proibito è l'unico film girato da Curzio Malaparte. Uscito nel 1951 s'iscrive nel periodo storico del Secondo dopoguerra e deve fare i conti con la corrente estetica dominante dell'epoca: il neorealismo. Attraverso un'analisi approfondita delle sequenze del film e una rilettura dei testi teorici di Malaparte sul cinema, l'articolo inquadra il film nel suo contesto storico, con lo scopo di definire la sua singolare concezione della regia e di collocare un'opera ambigua e manierista all'interno dei temi e delle ossessioni del suo autore.

Il Cristo Proibito (The Forbidden Christ) is the sole film by Curzio Malaparte. Released in 1951, it is in keeping with the historical period following World War II, and should be included in the dominant movement of the period, Neorealism. By studying the film sequences and rereading Malaparte's theoretical texts on cinema, this article makes an effort to reposition the film in this context, in order to clarify the original Malapartien approach to film direction, placing this mannerist and ambiguous work in the heart of the themes and obsessions of the writer-director.

\section{INDEX}

Parole chiave : Malaparte, Seconda guerra mondiale, neorealismo, espressionismo, tragedia, sacrificio, senso di colpa, De Santis

Keywords : Malaparte, World War II, Neorealism, Expressionism, tragedy, sacrifice, guilty feeling, De Santis

Mots-clés : Malaparte, Seconde Guerre mondiale, néoréalisme, expressionnisme, tragédie, sacrifice, culpabilité, De Santis

\section{AUTEUR}

\section{LAURENT SCOTTO}

Université Grenoble Alpes 